\title{
$\nabla$
}

\section{Doença de Kawasaki*}

\author{
Kawasaki disease
}

\author{
Patrícia Aparecida de Castro ${ }^{1}$ \\ Izelda Maria Carvalho Costa ${ }^{3}$
}

\author{
Lílian Mendes Ferreira Urbano ${ }^{2}$
}

Resumo: A doença de Kawasaki é vasculite sistêmica e aguda de etiologia desconhecida. Constitui a principal causa de doença cardíaca adquirida em crianças nos EUA. Ocorre mais frequentemente em meninos, $80 \%$ dos casos em crianças com menos de cinco anos, sendo rara após os oito anos. Pode atingir crianças de todas as raças, tendo maior incidência entre os descendentes asiáticos.

Caracteriza-se por febre, conjuntivite bilateral não exsudativa, eritema e edema de língua, lábios e mucosa oral, alterações de extremidades, linfonodomegalia cervical, exantema polimórfico. Aneurismas e estenoses de artérias coronárias são comuns em percentual que varia de 20 a $25 \%$ dos pacientes não tratados, podendo posteriormente levar a infarto agudo do miocárdio e morte súbita. O tratamento com imunoglobulina intravenosa é efetivo e deve ser iniciado precocemente a fim de evitar sequelas cardíacas. O desenvolvimento de testes diagnósticos, terapêuticas mais específicas e a prevenção dessa doença potencialmente fatal em crianças dependem dos contínuos avanços na determinação de sua etiopatogenia.

Palavras-chave: Aneurisma coronário; Aspirina; Exantema; Síndrome do linfonodo mucocutâneo

\begin{abstract}
Kawasaki disease is a systemic acute vasculitis of unknown etiology. It is the leading cause of acquired heart disease in children in the USA. It occurs more frequently in boys and eighty percent of the cases occur in children under five years of age. The disease rarely occurs after eight years and it can affect children of all races, with higher incidence among Asian descendants.

Kawasaki disease is characterized by fever, bilateral non-exudative conjunctivitis, redness and swelling of the tongue, lips and oral mucosa, abnormalities in the extremities, cervical lymph node, and polymorphic exanthema. Aneurysms and stenoses of coronary arteries occur in approximately 20 to $25 \%$ of untreated patients and subsequently can lead to acute myocardial infarction and sudden death. Treatment with intravenous immunoglobulin is effective and should be initiated early to prevent cardiac sequel. The development of diagnostic tests, more specific treatment approaches and prevention of this potentially fatal disease in children depends on continuous advances in the determination of its pathogenesis.

Keywords: Aspirin; Coronary aneurysm; Exanthema; Mucocutaneous lymph node syndrome
\end{abstract}

\section{INTRODUÇÃO}

Trata-se de vasculite aguda e multissistêmica que compromete vasos de médio calibre. ${ }^{1}$ É mais frequente em crianças, principalmente com menos de cinco anos, porém há relatos na literatura de casos em adultos. A doença de Kawasaki (DK) pode causar vasculite em vários órgãos, como pulmão, intestino, vesí- cula biliar, sistema nervoso central, entre outros, mas o comprometimento cardíaco é o mais significativo, com a formação de aneurismas coronarianos. O diagnóstico é essencialmente clínico, e o tratamento medicamentoso, logo que iniciado, conduz à melhora clínica e reduz os riscos de sequela cardíaca.

\footnotetext{
Aprovado pelo Conselho Editorial e aceito para publicação em 29.05.2009.

* Trabalho realizado no Ambulatório de Dermatologia Pediátrica do Serviço de Dermatologia do Hospital Universitário de Brasília (HUB) - Brasília (DF), Brasil. Conflito de interesse: Nenhum / Conflict of interest: None Suporte financeiro: Nenhum / Financial funding: None

Médica residente do terceiro ano do Departamento de Dermatologia da Universidade de Brasília (UnB) - Brasília (DF), Brasil. Médica residente do segundo ano do Departamento de Dermatologia da Universidade de Brasília (UnB) - Brasília (DF), Brasil.

Mestre e Doutora em dermatologia. Coordenadora do ambulatório de Dermatologia Infantil do Hospital Universitário de Brasília (HUB/UnB) e orientadora do curso de pós-graduação em ciências da saúde da Universidade de Brasília (UnB) - Brasília (DF), Brasil. 


\section{HISTÓRICO}

A DK foi inicialmente descrita na literatura médica por Tomisaku Kawasaki em 1967. ${ }^{2} \mathrm{Na}$ ocasião, foram relatados 50 casos acompanhados no período de 1961 a 1967.3 Ele acreditava tratar-se de doença benigna, autolimitada, que não deixava sequelas. Denominou a entidade síndrome do linfonodo mucocutâneo. ${ }^{4}$ Em 1970 ocorreram 10 mortes de crianças com menos de dois anos de idade vítimas da DK, revelando face mais agressiva da doença., ${ }^{4,5} \mathrm{O}$ patologista Noboru Tanaka descobriu uma trombose de artéria coronária durante a necrópsia de uma criança com diagnóstico prévio de $\mathrm{DK}^{4} \mathrm{O}$ pediatra Takajiro Yamamoto observou ritmo de galope e insuficiência cardíaca congestiva em um de seus pacientes com DK. Yamamoto e colaboradores publicaram 23 casos de DK dos quais 11(48\%) apresentavam alterações eletrocardiográficas, o que os levou à conclusão de ser o envolvimento cardíaco característica comum da síndrome. ${ }^{3}$ Em 1974, Kawasaki lançou a primeira publicação sobre a DK na língua inglesa." ${ }^{3,6}$ Melish, em 1976, relatou o primeiro caso de DK nos EUA. ${ }^{4}$

\section{Epidemiologia}

De ocorrência universal, ${ }^{7}$ a DK atinge todas as faixas etárias pediátricas, ainda que em $85 \%$ dos casos crianças com menos de cinco anos. ${ }^{6,7}$ É infrequente em pacientes com menos de seis meses ou mais de oito anos, casos em que, entretanto, há maior risco de formação de aneurismas coronarianos. ${ }^{7}$ A incidência da DK varia de uma parte do mundo para outra. É marcadamente mais prevalente no Japão e em crianças descendentes de japoneses, com incidência anual de aproximadamente 112 casos por 100.000 crianças com menos de cinco anos. ${ }^{4,6,8,9}$ Ocorreram três grandes epidemias no Japão, em 1979, 1982 e 1986. Sua incidência anual nos EUA varia de 9,1 a 32,5 casos por 100.000 crianças com menos de cinco anos, sendo mais comum nos americanos descendentes dos asiáticos e das ilhas do pacífico (32,5/100.000 crianças $<5$ anos), intermediária nos afro-americanos (16,9/100.000 crianças $<5$ anos) e hispânicos $(11,1 / 100.000$ crianças $<5$ anos), e menos frequente em brancos $(9,1 / 100.000$ crianças $<5$ anos $){ }^{8}$ É a causa mais comum de doença cardíaca adquirida em crianças nos EUA. ${ }^{5,6}$ A relação entre meninos e meninas varia de 1,5 a 1,7: 1. ${ }^{2,8} \mathrm{O}$ índice de recorrência da DK no Japão é de $3 \%$ e na América do Norte é de aproximadamente $1 \%{ }^{2,3}$

Um estudo da DK em famílias japonesas mostrou que a incidência do segundo caso da doença um ano depois do primeiro, na mesma família, é significativamente mais elevada do que na população geral. ${ }^{2,4}$

Aproximadamente 50\% dos segundos casos ocorrem nos primeiros $\mathbf{1 0}$ dias após o aparecimento do caso índice. ${ }^{3,4} \mathrm{O}$ risco de ocorrência em gêmeos é de $13 \%$.
Esses achados sugerem que a predisposição genética em interação com a possível exposição a agentes etiológicos e ambientais teriam papel na etiopatogenia da doença, que é mais comum nos meses de inverno e primavera. ${ }^{4}$

\section{Etiopatogenia}

A causa da doença de Kawasaki permanece desconhecida apesar de as características clínicas (doença febril autolimitada) e epidemiológicas (sazonalidade e caráter epidêmico) favorecerem a hipótese de um agente infeccioso ser o determinante causal, que, entretanto, ainda não está comprovada.

Existem teorias em estudo, ainda com discordâncias no meio científico. Uma delas é a teoria do coronavírus NL-63; em 2005 pesquisadores de New Haven, Connecticut, relataram a presença de um coronavírus humano (HCoV) detectado, por meio de reação em cadeia da polimerase (PCR), em secreções respiratórias de oito crianças do total de 11 pacientes com doença de Kawasaki e apenas em um dos 22 pacientes do controle. ${ }^{10}$

Não obstante, outros cinco estudos posteriores não encontraram o coronavírus em amostras de tecido respiratório ou nasofaríngeo de doentes com DK com significância estatística, o que invalida a possibilidade de esse vírus ser o agente causal da doença. ${ }^{11}$

A outra teoria aventada é a da estimulação imunológica por superantígenos bacterianos tais como as toxinas estafilocócicas e estreptocócicas. ${ }^{12} \mathrm{O}$ superantígeno liga-se, intacto, sem que tenha sido processado, à célula apresentadora de antígeno por meio do complexo de histocompatibilidade maior de classe II (MHC classe II) e ao domínio V, do receptor de linfócito $\mathrm{T}$ (TcR) em sítios diferentes daqueles de antígenos comuns e desencadeia ativação imunológica exacerbada com produção e liberação de citocinas pró-inflamatórias em concentração excessiva, tais como o fator de necrose tumoral alfa (TNF-alfa), interleucina 1(IL-1), IL-2, entre outras. ${ }^{11,13}$ Apresenta ainda as funções de ativar as células natural killer, ativação policlonal de linfócitos $\mathrm{B}$, acentuação de atividade da endotoxina e efeito tóxico sobre o endotélio. ${ }^{13-15}$ No entanto, um estudo multicêntrico não mostrou diferenças no isolamento de bactérias produtoras de superantígenos entre pacientes com DK e o grupo-controle febril. ${ }^{16}$

A terceira teoria é a da resposta oligoclonal IgA na doença de Kawasaki aguda. Análise de sequência gênica da cadeia pesada alfa de imunoglobulinas provenientes de infiltrado inflamatório da parede arterial de pacientes com DK mostrou que um número restrito de anticorpos específicos imunoglobulinas A (IgA) estava presente (resposta oligoclonal), indicando resposta imune antígenodirigida. ${ }^{17} \mathrm{Com}$ o objetivo de 
detectar o antígeno determinante dessa resposta oligoclonal, anticorpos sintéticos foram produzidos in vitro após clonagem de genes das regiões variáveis $\alpha$ e $\kappa$ das imunoglobulinas presentes na parede arterial inflamada de pacientes com DK. As imunoglobulinas sintéticas IgA detectaram inclusões citoplasmáticas no epitélio ciliar brônquico de crianças com DK. Os estudos indicam que tais inclusões são mais consistentes com agregados de proteínas virais e associadas com ácido nucleico. ${ }^{18}$ Inclusões citoplasmáticas têm sido identificadas em pacientes asiáticos e não asiáticos do Japão e Estados Unidos com doença de Kawasaki por meio dos anticorpos monoclonais sintéticos, corroborando essa teoria da resposta por IgA e a possibilidade de a DK ser resultado de um agente infeccioso ainda não determinado. ${ }^{11,18}$

$\mathrm{O}$ aumento da frequência de DK entre asiáticos e seus descendentes, assim como a incidência elevada entre familiares de doentes favorecem a ideia da predisposição genética na doença de Kawasaki. ${ }^{19}$ Um estudo revelou 67 genes distintos associados à doença de Kawasaki e envolvidos na função endotelial, metabolismo lipídico, adesão plaquetária e ativação imune. ${ }^{20}$ Alguns alelos gênicos (dos gens CCR3, CCR2, CCR5 que são receptores de citocinas) mostram associação significativa com doença de Kawasaki, o que sugere a susceptibilidade genética. ${ }^{21}$

O polimorfismo do gen do receptor de interleucina 1 (IL-1R) também tem sido implicado na patogênese da doença de Kawasaki. ${ }^{22}$

Há vários estudos buscando elucidar mais detalhadamente a patogênese e, por meio desse conhecimento, encontrar terapêuticas mais direcionadas e medidas preventivas contra as sequelas cardíacas. Estudos mostram que níveis elevados de fator de necrose tumoral alfa (TNF- $\alpha$ ) são significativos em vasos coronarianos daqueles pacientes com lesão cardíaca, o que pode implicar terapêutica mais específica (antiTNF alfa) em casos de comprometimento cardíaco ou refratariedade ao tratamento. ${ }^{23}$

As metaloproteinases de matriz (MMP) são endopeptidases zincodependentes envolvidas na degradação de matriz extracelular como as fibras de colágeno e elásticas. Essas enzimas são produzidas pelas células musculares lisas da parede arterial durante resposta inflamatória e sofrem up-regulation pelo fator de necrose tumoral alfa. Estudos evidenciam que a MMP-9 está relacionada ao dano vascular coronariano. ${ }^{24}$ Já o polimorfismo gênico da MMP-13 (certas frequências genotípicas) apresenta associação significativa com a formação de aneurismas coronarianos. ${ }^{25}$

Há uma família de proteínas (S100) liberadas por neutrófilos e monócitos durante a cascata inflamatória que é responsável pela migração de células inflamatórias através da parede vascular; age ligando-se a receptores nas células endoteliais (chamados RAGE). Existe ainda o fator inibitório (s-RAGE) do receptor RAGE que impede a ligação da proteína S100A12 a seu receptor, equilibrando a resposta inflamatória. No entanto, na fase aguda da doença, o nível sérico do sRAGE encontra-se diminuído principalmente naqueles pacientes que não respondem ao tratamento. Sabe-se ainda que os níveis de s-RAGE correlacionamse negativamente com os da proteína pró-inflamatória S100A12. ${ }^{26,27}$

A hipótese etiopatogênica mais aceitável é a de que a doença de Kawasaki seja causada por um agente infeccioso ainda não identificado, que determina alterações clínicas em indivíduos susceptíveis geneticamente, no caso os asiáticos.

\section{Patologia}

As alterações histológicas encontradas na DK consistem em vasculite sistêmica generalizada, afetando predominantemente vasos de médio calibre com predileção pelas artérias coronárias. ${ }^{1-4,6}$ Alterações inflamatórias sistêmicas podem ser observadas em vários órgãos causando miocardite, pericardite, vasculites, meningite asséptica, pneumonite, linfadenite e hepatite. ${ }^{4,6}$

Nos estádios iniciais da vasculite ocorre edema das células endoteliais com degeneração nuclear acompanhado de edema e inflamação da camada adventícia. ${ }^{4}$ A lâmina elástica interna permanece intacta. $^{2}$ Verifica-se infiltrado inflamatório neutrofílico inicialmente (nos primeiros sete a nove dias), com rápida transição para mononuclear com predomínio de linfócitos T citotóxicos CD8+ e imunoglobulina IgA.,

Nas artérias coronárias são observadas alterações inflamatórias na camada média com edema e necrose de células musculares; posteriormente há progressão do processo inflamatório, que passa a envolver todo o vaso., ${ }^{3,4}$ Nesse estádio, ocorre destruição das lâminas elásticas interna e externa, tornando as camadas dos vasos acometidos indistinguíveis. ${ }^{2}$ Com a perda da integridade estrutural, ocorre a formação dos aneurismas. ${ }^{4}$ Ocorre também a proliferação de fibroblastos. As metaloproteinases de matriz (MMP) têm importante papel no processo de remodelação arterial. ${ }^{2,3}$ Com a remodelação, podem ocorrer estenoses, calcificações e formação de trombos. A atividade inflamatória permanece durante período que varia de semanas a meses com fibrose progressiva. ${ }^{3}$

\section{Diagnóstico Clínico e laboratorial}

$\mathrm{Na}$ ausência de teste diagnóstico específico ou achado clínico patognomônico para a DK, pesquisadores, com base em estudos epidemiológicos, estabeleceram critérios clínicos para facilitar esse diagnóstico (Quadro 1). ${ }^{2,7,28,29}$ A presença de febre persistente durante cinco dias ou mais, associada a quatro dos 
QuAdro 1: Critérios diagnósticos da doença de Kawasaki segundo a American Heart Association

- Febre por cinco dias ou mais

- Conjuntivite não purulenta

- Língua framboesiforme, eritema e edema de orofaringe, fissuras e eritema labial

- Eritema e edema de mãos e pés com descamação periungueal

- Exantema escarlatiniforme, morbiliforme ou polimórfico

- Linfonodomegalia cervical

outros cinco critérios clínicos, fecha o diagnóstico de DK. Não existem testes diagnósticos específicos para a DK, mas existem achados laboratoriais característicos (Quadro 2).

Alguns pacientes são diagnosticados como portadores de DK atípica ou incompleta uma vez que não preenchem todos os critérios diagnósticos propostos. ${ }^{2,29,30} \mathrm{O}$ termo mais apropriado é DK incompleta, pois esses pacientes apresentam alguns dos sintomas típicos da doença e não manifestações atípicas. A DK incompleta deve ser considerada em todas as crianças com febre inexplicada por mais de cinco dias associada a dois ou três dos principais achados clínicos da DK. ${ }^{1,30} \mathrm{~A}$ forma incompleta é mais frequente em crianças com menos de seis meses. ${ }^{3} \mathrm{O}$ diagnóstico da DK incompleta é baseado em achados ecocardiográficos de alterações nas artérias coronárias; mesmo naqueles pacientes com menos de três critérios diagnósticos, porém com lesão coronariana, o tratamento é indicado com imunoglobulina intravenosa. Os critérios diagnósticos convencionais propostos pela American Heart Association são falhos para reconhecer a forma incompleta da doença. 5, 32,33 $^{2}$

O eletrocardiograma pode ser normal ou mostrar arritmias, prolongamento do intervalo PR ou alterações não específicas no segmento ST e na onda $T^{2,8}$ A radiografia de tórax pode ser geralmente normal, mas em $14,7 \%$ dos casos são encontradas alterações como padrão de infiltrado reticulogranular ou peribrônquico, derrame pleural ou atelectasia. Tais anormalidades surgem após 10 dias de doença e mostram resolução radiográfica 10 a 50 dias depois do início do quadro. ${ }^{6}$

\section{Quadro clínico}

$\mathrm{Na}$ doença de Kawasaki a febre, sinal característico da fase aguda da doença, é geralmente alta (acima de $39^{\circ} \mathrm{C}-40^{\circ} \mathrm{C}$ ), remitente e acompanhada de extrema irritabilidade ${ }^{34,35} \mathrm{O}$ primeiro dia de febre é considerado o primeiro dia de doença, no entanto, alguns pacientes ocasionalmente apresentam outras manifestações clínicas antecedendo o quadro febril. ${ }^{3,4}$ A duração da febre é, em média, de uma a duas semanas, podendo, na ausência de tratamento, estender-se até três ou quatro semanas. Responde parcialmente ao uso de antipiréticos, mas não cessa com a introdução de antibióticos. Ao se iniciar, porém, a terapêutica apropriada - imunoglobulina intravenosa (IGIV) e aspirina - a febre cessa em dois dias., ${ }^{2,36}$

A conjuntivite bilateral não exsudativa envolve principalmente a conjuntiva bulbar em relação às conjuntivas palpebral e tarsal, é indolor e ocorre na fase aguda da doença. Iridociclite pode acompanhar o quadro clínico com rápida resolução e raramente associada à fotofobia. ${ }^{2,3,6,8}$

As alterações vistas na semimucosa labial são caracterizadas por eritema, edema com fissuras, descamação e exsudação (Figura 1); a mucosa orofaríngea apresenta-se com enantema, e a língua com aspecto framboesiforme (eritema intenso com papilas gustativas proeminentes). Ulcerações e exsudato faríngeo não ocorrem. ${ }^{2,4}$

$\mathrm{Na}$ fase aguda da doença também são descritos o eritema e/ou induração de regióes palmar e plantar

\section{QuADro 2: Achados laboratoriais na doença de Kawasaki}

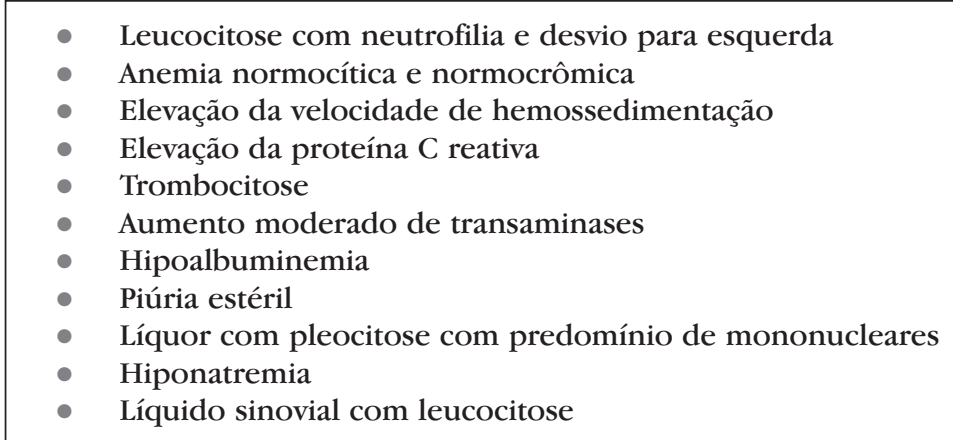




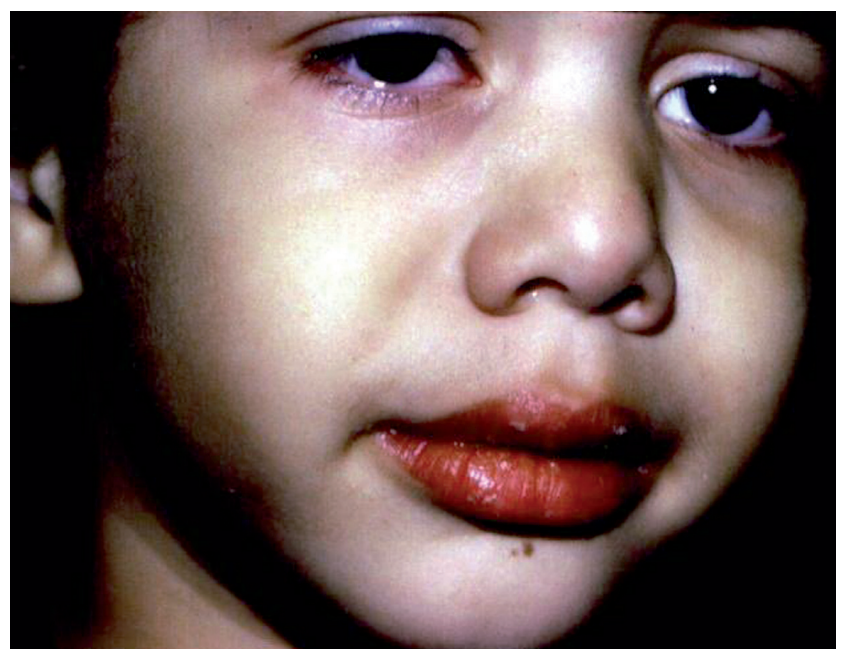

Figura 1: Aspecto típico da DK em criança de dois anos de idade com vermelhidão, ressecamento e sangramento dos lábios mais hiperemia conjuntival bilateral

acompanhados de aumento da sensibilidade dolorosa; após duas ou três semanas de doença - fase subaguda - inicia-se a descamação periungueal dos pés e mãos, que progride para palmas e plantas (Figura 2). ${ }^{3,8}$ Um ou dois meses após instalado o quadro, podem surgir linhas de Beau nas unhas afetadas. ${ }^{2}$

O rash cutâneo que surge no paciente com doença de Kawasaki é não específico, polimórfico, não pruriginoso e geralmente aparece até o quinto dia de febre. O exantema cutâneo pode compreender lesões maculopapulares eritematosas e difusas, a forma mais comum, além de rash tipo urticariforme, escarlatiniforme, eritrodérmico, purpúrico, eritema multiforme-like ${ }^{7} \mathrm{e}$, mais raramente, micropústulas em superfície extensoras de membros. ${ }^{37,38}$ Não há relatos na literatura de lesões vesicobolhosas. O rash compromete principalmente o tronco e extremidades, com predileção pelas regiões inguinal e perineal, que apresentam, no decorrer da evolução clínica, descamação ainda na fase aguda da doença (Figura 3). ${ }^{2-4,6}$ A linfadenopatia cervical é o critério diagnóstico mais incomum, ocorrendo em percentual que varia de 50 a $75 \%$ dos pacientes, ao passo que os demais critérios clínicos são vistos em $90 \%$ dos casos. É geralmente unilateral e localizada no triângulo cervical anterior. Conforme definição dos critérios diagnósticos, deve haver mais de um linfonodo comprometido e com diâmetro superior a $1,5 \mathrm{~cm}$. Os linfonodos afetados são indolores ou pouco dolorosos, não flutuantes e não supurativos; o eritema da pele suprajacente pode ocorrer. Devese atentar para aquelas crianças com febre e adenite cervical que não respondem aos antibióticos, pois a doença de Kawasaki deve compor os diagnósticos diferenciais. ${ }^{2,6,7}$

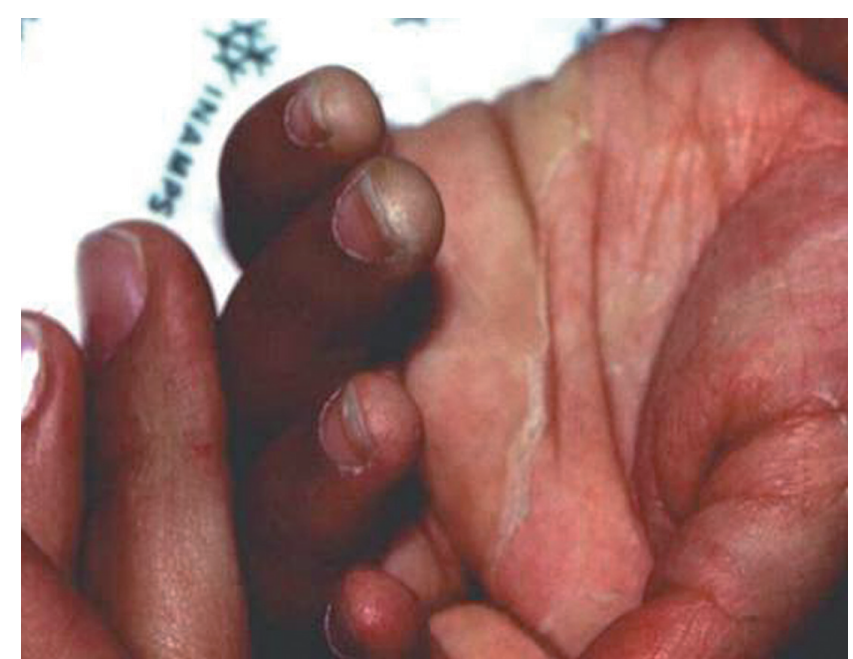

Figura 2: Presença de descamação em extremidades com progressão para região palmar na fase subaguda da doença

A doença de Kawasaki é dividida em três fases clínicas: aguda, subaguda e de convalescença. A fase aguda, que dura de uma a duas semanas, compreende febre e os demais critérios diagnósticos, além de achados clínicos associados, como miocardite, derrame pericárdico, meningite asséptica (10-25\%), diarreia (15\%), disfunção hepática (5\%), uveíte (17\%) e artrite e/ou artralgia (30\%). A fase subaguda inicia-se quando a febre, o rash e a linfadenopatia apresentam resolução, uma ou duas semanas depois do início da doença, porém há irritabilidade, anorexia e conjuntivite. Nessa fase, cuja duração é de cerca de quatro semanas, ocorrem descamação periungueal, trombocitose, formação de aneurismas coronarianos, e o risco de morte súbita é maior. Complicações neurológicas podem surgir em $1 \%$ dos casos e incluem parali-

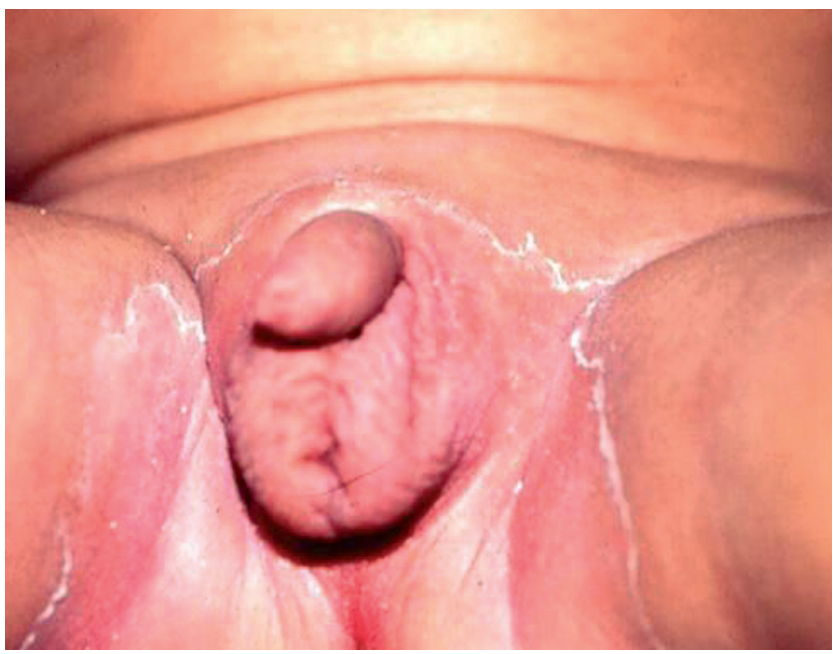

Figura 3: Descamação perineal na fase aguda da doença 
sia de nervo facial, ataxia, encefalopatia, hemiplegia e infarto cerebral. A fase de convalescença começa quando os sinais clínicos desaparecem e vai até a normalização da velocidade de hemossedimentação (VHS), durando, usualmente, de seis a oito semanas após início do quadro febril. ${ }^{4,6,39}$ A ocorrência da doença em adultos é incomum, havendo aproximadamente 60 casos relatados na literatura com idade variando de 18-30 anos e maior incidência de relatos na Europa, sem registros de morte em adultos na fase aguda. ${ }^{39-41}$ Os critérios diagnósticos foram estabelecidos para crianças e não são validados para adultos, porém são utilizados na prática clínica.

Seve e colaboradores, ${ }^{41}$ após estudo de 57 casos em adultos, estabeleceram as diferenças nas apresentações clínicas entre crianças e adultos: estes têm mais acometimento de linfonodos cervicais (93\% dos adultos versus $15 \%$ das crianças), hepatite (65\% versus $10 \%$ ) e artralgia (61\% versus $24-38 \%$ ). No entanto, os adultos são menos frequentemente afetados por meningite ( $10 \%$ dos adultos versus $34 \%$ das crianças), trombocitose (55\% versus $100 \%$ ) e aneurismas de artérias coronárias (5\% versus $18-25 \%$ ). A conclusão diagnóstica de doença de Kawasaki no adulto é difícil de ser confirmada, e deve-se levar em consideração um conjunto de informações como ausência de infecção, não melhora clínica com antibióticos e presença dos critérios diagnósticos, como feito na criança.

Diversas manifestações clínicas decorrentes da vasculite sistêmica estabelecida podem ser descritas na doença de Kawasaki; são, entretanto, achados secundários e não compõem os critérios diagnósticos (Quadro 3). ${ }^{2-4,6,8,42}$ A síndrome hemofagocítica, que é caracterizada por febre persistente, citopenias, hepatoesplenomegalia, disfunção hepática secundárias à ativação excessiva de macrófagos, é complicação rara e grave da doença de Kawasaki que parece ocorrer em pacientes com febre recorrente ou refratários ao tratamento. ${ }^{43}$

\section{Manifestações cardiovasculares e exames de imagem}

As manifestações cardíacas podem ser exacerbadas na fase aguda determinando aumento na mortalidade e morbidade a longo prazo. A doença de Kawasaki é a maior causa de doença cardíaca adquirida na infância nos EUA e Japão, e importante causa naqueles adultos em que a incidência da doença é comum. Pode haver miocardite, pericardite, endocardite, comprometimento valvular e coronariano com repercussão hemodinâmica. O exame clínico da criança com alteração cardíaca pode revelar precórdio hiperdinâmico com taquicardia, sopro pansistólico em caso de regurgitação mitral significativa e ritmo de galope devido à instalação de insuficiência cardíaca. ${ }^{2,6}$
A miocardite é detectada em 50-70\% dos pacientes por meio de exames com radioisótopos. A severidade da inflamação miocárdica parece não estar relacionada ao risco de formação de aneurismas coronarianos. Embora a maioria das crianças apresente contratilidade miocárdica anormal à ecocardiografia na fase aguda, a função cardíaca é logo restituída com a introdução da IGIV. ${ }^{44}$

O dano coronariano, que pode variar desde dilatação e estenose até formação de aneurisma, ainda ocorre em $5 \%$ dos pacientes adequadamente tratados e em $20-25 \%$ daqueles não tratados. ${ }^{2,6,45}$ A frequência de envolvimento coronariano é maior nos lactentes com menos de seis meses em relação àqueles de seis a 12 meses de idade. A dilatação coronariana, quando ocorre, pode ser evidenciada pela ecocardiografia bidimensional por volta do décimo dia de doença, e o pico de ocorrência da dilatação ou aneurisma é com quatro semanas de doença. Em 1\% dos casos ocorre formação de aneurismas gigantes. ${ }^{3,4}$ Os aneurismas fusiformes e saculares aparecem de 18 a 25 dias depois de instalada a doença. Um estudo de 598 crianças com Kawasaki mostrou o envolvimento da artéria coronária esquerda em $12 \%$ dos casos; em $3 \%$ da artéria coronária direita; e de ambas em $8 \% .{ }^{46}$

Conforme a classificação da American Heart Association, ${ }^{2}$ os aneurismas são classificados em pequenos (diâmetro interno da parede do vaso $<5 \mathrm{~mm}$ ), médios (diâmetro variando de cinco a $8 \mathrm{~mm}$ ) e gigantes (diâmetro $>8 \mathrm{~mm}$ ). Geralmente os aneurismas pequenos e fusiformes tendem à regressão, enquanto os gigantes e saculares têm prognóstico reservado com risco de trombose e estenose ${ }^{42,47}$ Os aneurismas podem ser formados, menos frequentemente, em outras artérias como subclávia, braquial, axilar, ilíaca ou femoral e até em aorta abdominal e renal. ${ }^{3}$

Há evidências de que existem fatores de risco relacionados à formação de aneurismas coronarianos, tais como febre recorrente a despeito do uso de IGIV, VHS aumentado, anemia e hipoalbuminemia, sexo masculino, idade inferior a um ano, hiponatremia e trombocitopenia. ${ }^{48}$

Em virtude dos riscos de sequelas cardíacas decorrentes da doença de Kawasaki, o monitoramento cardiovascular por meio de exames de imagem é imprescindível.

O exame considerado primordial, não invasivo, com alta sensibilidade e especificidade para detectar anormalidades nos segmentos proximais das artérias coronarianas é a ecocardiografia (nível de evidência C). O ecocardiograma é fundamental na avaliação de crianças com febre e alguns dos achados da DK. O exame pode mostrar brilho perivascular, ectasia ou estreitamento das artérias coronárias que representam a arterite coronariana antes da formação do aneu- 
QuADro3: Achados clínicos secundários da doença de Kawasaki

Aparelho cardiovascular

- Miocardite, pericardite, regurgitação valvular, aneurismas de artérias coronárias

Aparelho musculoesquelético

- Poliartrite e/ou artralgia

- Rabdomiólise

Aparelho gastrointestinal

- Diarreia, dor abdominal, vômitos, disfunção hepática, pancreatite, vesícula hidrópica, colangite, intussuscepção, pseudo-obstrução intestinal, ascite, infarto esplênico

\section{Aparelho pulmonar}

- Sintomas influenza-like, derrame pleural

- Infiltrado pulmonar reticulogranular

- Atelectasia

Aparelho geniturinário

- Uretrite, prostatite, cistite, priapismo, insuficiência renal aguda, nefrite intersticial, orquite, síndrome nefrótica

\section{Sistema nervoso central}

- Meningite asséptica

- Surdez neurossensorial

Pele e anexos

- Eritema e induração em sítio de vacinação de BCG

- Linhas de Beau

- Gangrena de dedos

Fonte: Newburger JW, ${ }^{2}$ Kim DS, ${ }^{3}$ Rowley AH, ${ }^{4}$ Chung CJ, ${ }^{6}$ Satou GM, ${ }^{8}$ Pinna GS ${ }^{42}$

risma, que raramente é visto antes de 10 dias de doença. Redução da contratilidade miocárdica do ventrículo esquerdo, regurgitação da valva mitral e efusão pericárdica são achados ecocardiográficos frequentes em pacientes com DK na fase aguda.

Para aqueles casos sem comprometimento cardíaco, o ecocardiograma deve ser realizado no momento do diagnóstico, na segunda semana e entre a sexta e a oitava semanas do início do quadro.

É importante reconhecer as limitações do ecocardiograma em visualizar trombose e estenose em segmentos distais das coronárias. ${ }^{2,42}$ Há outras opções diagnósticas, como ressonância nuclear magnética (RNM), angioressonância, tomografia computadorizada de alta resolução e angiografia, que são utilizadas conforme disponibilidade, vantagens, gravidade e indicações de cada caso. Tanto a RNM quanto a angiorressonância são capazes de detectar aneurismas em ramos distais das coronárias, podendo a última ser útil em estágios precoces da doença mesmo em crianças com menos de seis meses. ${ }^{49}$ Recentemente tem sido recomendada a tomografia computadorizada de alta resolução, que apresenta vantagens em relação à ecocardiografia transtorácica ou RNM, detectando calcificação na parede arterial, fazendo rápida coleta de imagens e simplificando sua interpretação, enquanto a RNM necessita de prolongamento do tempo anestésico das crianças para captura de imagens. ${ }^{2,50,51} \mathrm{O}$ objetivo de tais exames é primordialmente detectar as alterações cardíacas a fim de estabelecer uma estratificação de risco para adequado manejo terapêutico. ${ }^{51}$

A angiografia é o exame padrão-ouro para avaliar o comprometimento cardíaco, porém é teste invasivo e com riscos inerentes, tendo suas indicações de uso. Em pacientes com aneurismas médios ou fusiformes visualizados à ecocardiografia e com tendência à regressão não há indicação para esse exame. As indicações da angiografia dependem das alterações vistas nos exames não invasivos como sinais de isquemia miocárdica ao ecocardiograma de estresse, falha em visualizar ramos distais das coronárias, múltiplos aneurismas, aneurisma gigante, estenose coronariana e acompanhamento pós-revascularização miocárdica (nível C de evidência). ${ }^{6} \mathrm{O}$ momento ideal para realização do exame varia de acordo com o centro hospitalar, sendo, entretanto, recomendado entre seis e 12 
QuADro 4: Diagnósticos diferenciais da doença de Kawasaki

\begin{tabular}{|l|}
\hline Síndrome de Stevens Johnson \\
- \\
- Artrite reumatoide juvenil \\
- Síndrome da pele escaldada estafilocócica \\
- Líndrome do choque tóxico \\
- Intoxicação por mercúrio \\
- Esarampo \\
- Eebre maculatina \\
Linfadenites cervicais bacterianas \\
\hline
\end{tabular}

meses depois do início da doença e após resolução do processo inflamatório agudo (nível de evidência C).

Uma revisão estimou a incidência de infarto agudo do miocárdio em gestantes em 1: $10.000 .^{52}$ Há poucos estudos acerca de gestação e trabalho de parto em grávidas com doença coronariana por Kawasaki. Em estudo de 13 gestantes com doença de Kawasaki na infância e lesão coronariana, nenhuma apresentou trombose coronariana fazendo uso de baixas doses de aspirina, o que foi considerado seguro para a gravidez e o parto. Nove gestantes tiveram parto vaginal com anestesia epidural sem complicações cardíacas. É indicada analgesia no parto para gestantes com doença cardíaca, pois mantém a função hemodinâmica e diminui o trabalho cardíaco. Naqueles casos sintomáticos, pode ser avaliada a possibilidade de parto cesáreo. ${ }^{53}$

\section{Diagnósticos diferenciais}

Os principais diagnósticos diferenciais encontram-se no quadro ${ }^{4.4}$

As alterações laboratoriais e peculiaridades clínicas devem auxiliar na exclusão diagnóstica de cada doença. Um importante diagnóstico diferencial para o dermatologista é a escarlatina, devido a semelhanças clínicas. Ambas apresentam exantema com descamação palmoplantar, enantema de mucosa oral com língua framboesiforme e linfonodomegalia. Não obstante, a escarlatina revela faringite exsudativa causada por estreptococos do grupo A e boa resposta clínica em 24-48 horas do início da antibioticoterapia.

Há também o eritema perineal toxinomediado recorrente, que é doença cutânea mediada por superantígenos produzidos por estreptococos e estafilococos, e clinicamente semelhante à doença de Kawasaki. Nele se observam eritema perineal com descamação, enantema de mucosa oral com língua framboesiforme e eritema palmoplantar descamativo, sinais comuns à doença de Kawasaki. O diagnóstico diferencial, porém, baseia-se na ausência de febre, exantema, con- juntivite e linfonodomegalia no eritema perineal toxinomediado. $^{54}$

\section{Evolução e prognóstico}

O curso evolutivo das lesões coronarianas na doença de Kawasaki é modificado temporalmente. Resolução angiográfica após um ou dois anos a partir do início do quadro é observada em percentual que varia de 50 a $67 \%$ dos vasos com aneurismas coronarianos. ${ }^{2,46}$ Há alguns fatores associados à possibilidade de regressão do aneurisma, como idade inferior a um ano, aneurismas pequenos e médios, morfologia fusiforme e aneurismas localizados em segmentos distais das coronárias. Os aneurismas podem evoluir desfavoravelmente com persistência destes, estenose, oclusão ou tortuosidades.

Enquanto os aneurismas tendem à regressão na maior parte dos casos, as estenoses, que representam a proliferação das camadas íntimas e média, são progressivas. Os aneurismas gigantes e persistentes evoluem mais frequentemente com estenose.

Kato e colaboradores ${ }^{46}$ relataram, em estudo prospectivo, a presença de estenose coronariana em pacientes com aneurisma persistente após período de 10 a 21 anos da fase aguda da doença. $\mathrm{O}$ infarto agudo do miocárdio (IAM) causado pela oclusão trombótica do aneurisma ou a estenose coronariana são as principais causas de morte na doença de Kawasaki. ${ }^{55} \mathrm{O}$ alto risco de IAM existe principalmente no $1^{\circ}$ ano após a doença, e a maioria de casos fatais devem-se à obstrução da artéria coronária esquerda ou ambas, tanto coronária direita como a artéria descendente anterior (ramo da artéria coronária esquerda). Naqueles pacientes com doença coronariana significativa há necessidade de realização de ecocardiograma de estresse para avaliar função miocárdica de modo a decidir acerca da indicação de angiografia e intervenção cirúrgica. ${ }^{2,46}$

O IAM em crianças tem apresentação clínica diferente da que se refere a adultos. Uma revisão de 
195 casos de IAM devido a Kawasaki em crianças hospitalizadas no Japão mostrou que os principais sintomas são hipotensão, vômitos, indisposição e dor abdominal; a dor torácica ocorreu mais frequentemente em crianças maiores. ${ }^{4}$ Em $37 \%$ dos casos não houve sintomas prévios. Esses achados realçam a importância da suspeição diagnóstica de IAM em crianças com Kawasaki ou doença prévia, ou ainda doença coronariana com epidemiologia positiva para DK, pois, dessa forma, uma intervenção precoce e correta pode diminuir a mortalidade nesse segmento.

As implicações a longo prazo dos aneurismas que regridem são desconhecidas. As artérias comprometidas apresentam menor distensibilidade e vasodilatação reduzida à infusão de dinitrato de isossorbida em relação àqueles vasos sanguíneos que não foram afetados pela doença. ${ }^{57,58} \mathrm{O}$ ecocardiograma revela áreas, em que os aneurismas sofreram regressão, com espessamento irregular da camada íntima-média. $O$ exame anatomopatológico evidencia tal espessamento da parede do vaso, apesar de o lúmen arterial ser normal. Ainda é incerta a associação de doença de Kawasaki com maior progressão da aterosclerose em adultos com doença prévia. ${ }^{4,59}$ Há relatos de casos de adultos jovens com doença isquêmica coronariana consistente com sequela de Kawasaki.

Há alguns fatores que sugerem um pior prognóstico, com risco de formação de aneurismas, quais sejam: duração da febre por mais de 16 dias, recorrência da febre após pelo menos 48 horas afebril, arritmias, sexo masculino, menores de um ano, presença de cardiomegalia e alterações laboratoriais como plaquetopenia, anemia e hipoalbuminemia no início do quadro. ${ }^{4}$

Alguns pesquisadores têm estudado a fisiologia coronariana naqueles pacientes com DK e sem lesões cardíacas. Essas crianças apresentam fluxo miocárdico menor e maior resistência das artérias coronarianas quando comparadas ao grupo-controle no estudo de Muzik e colaboradores. ${ }^{60}$

Em virtude do provável risco de maior morbidade e mortalidade em adultos com DK prévia, é recomendado que todas as crianças (incluídas aquelas sem envolvimento cardiovascular) sejam acompanhadas a cada período de três a cinco anos por meio de exames. A frequência do seguimento das crianças com anormalidades de artérias coronárias deve ser mais periódica e varia de caso a caso. Esses quadros demandam acompanhamento com o cardiopediatra e realização de exames de rotina (eletrocardiograma e ecocardiograma) acrescidas de testes de esforço e exames de perfusão miocárdica para as crianças com mais de 10 anos de idade. Caso o teste de esforço seja compatível com isquemia miocárdica, a angiografia coronariana está indicada. ${ }^{8}$

Nakamura e colaboradores ${ }^{61}$ elaboraram um estudo que, após seguimento de 6.576 pacientes com
DK por período de 12 a 22 anos, evidenciou taxa de mortalidade maior para os homens com sequela cardíaca, enquanto mulheres nessa condição e homens e mulheres sem sequelas cardíacas não tiveram aumento da taxa de mortalidade em relação à população geral. $O$ índice de prevalência da morbidade é de $0,57 \%$, sendo em homens $(0,63 \%)$ maior do que em mulheres $(0,32 \%){ }^{62}$

No Japão foi realizado estudo com 9.965 calouros da Universidade de Tóquio em que os estudantes e pais responderam a um questionário direcionado para identificar a DK previamente, ou então, fazia-se o diagnóstico pelo relato espontâneo dos pais de doença de Kawasaki na infância. Os resultados evidenciaram que é incerta a declaração espontânea dos pais sobre a ocorrência da doença e que a avaliação médica e direcionada detecta pacientes com Kawasaki previamente; o estudo sugere que um em cada 200 estudantes no Japão pode ter tido a doença e há necessidade de reconhecimento de tal fato em virtude dos riscos de sequelas cardíacas e morte. ${ }^{62}$

$\mathrm{O}$ índice de letalidade no Japão é de $0,8 \% .{ }^{4}$ Nos EUA a mortalidade intra-hospitalar dos pacientes com DK é de aproximadamente $0,17 \%$. As mortes são decorrentes das sequelas cardíacas. O pico de mortalidade ocorre entre 15 e 45 dias após o início da febre; muitos casos, porém, aparecem tardiamente por infarto agudo do miocárdio secundário à trombose coronariana em áreas de aneurisma ou, mais raramente, por ruptura de aneurismas.

\section{TRATAMENTO}

O tratamento da doença de Kawasaki na fase aguda visa reduzir a resposta inflamatória na parede da artéria coronária e prevenir a vasculite com suas consequências (trombose e aneurisma), enquanto a terapêutica nas fases subaguda e de convalescença objetiva prevenir a isquemia miocárdica e o infarto. ${ }^{3}$

A imunoglobulina intravenosa (IGIV) é o principal medicamento na doença de Kawasaki, sendo utilizada na fase aguda, preferencialmente nos primeiros sete a 10 dias da doença, a fim de diminuir a prevalência de anormalidades das artérias coronárias e abreviar a duração dos sintomas clínicos. ${ }^{42,63-66}$ Pode ainda normalizar mais rapidamente as proteínas inflamatórias de fase aguda - mas não a velocidade de hemossedimentação (VHS) - e melhorar a função miocárdica., 66

Em estudo retrospectivo recente concluiu-se que o início tardio da IGIV, após o oitavo dia de doença, reduz as chances de sucesso terapêutico. ${ }^{67}$ Os mecanismos de ação da IGIV permanecem desconhecidos; várias teorias têm sido aventadas para os possíveis mecanismos como supressão de macrófagos e monócitos ativados, bloqueio da interação entre o endotélio e as células natural killers, estimulação de 
receptores inibitórios, modulação de produção de citocinas, neutralização de superantígenos bacterianos, diminuição da síntese de anticorpos e aumento da atividade de linfócitos T supressores. ${ }^{68,69}$

Os pacientes devem ser tratados com IGIV na dose de $2 \mathrm{~g} / \mathrm{kg}$ em infusão única durante período variável de 10 a 12 horas (nível de evidência $A$ ) juntamente com ácido acetilsalicílico (AAS) na dose de 80$100 \mathrm{mg} / \mathrm{kg}^{2,3} \mathrm{~A}$ infusão da IGIV antes do quinto dia de doença não previne as sequelas cardíacas mais do que o tratamento feito do quinto ao sétimo dia de doença; essa infusão precoce aumenta a necessidade de retratamento. ${ }^{70,71}$

A IGIV pode acarretar alguns efeitos colaterais menores como sintomas vasomotores: cefaléia, febre e calafrios, hipotensão, além de outras alterações transitórias como leucopenia, neutropenia ou proteinúria, podem surgir. ${ }^{3,42,72}$ Raramente ocorrem complicações severas como meningite asséptica, trombose, choque anafilático e insuficiência renal aguda. Também são incomuns os efeitos adversos dermatológicos, quais sejam prurido, dermatite eczematosa, alopecia e eritema multiforme. ${ }^{72}$

As vacinações com vírus vivo atenuado (sarampo, rubéola, caxumba e varicela) devem ser prorrogadas por, pelo menos, 11 meses após a administração da IGIV devido à redução da imunogenicidade pelos anticorpos passivos do medicamento. ${ }^{73}$

A aspirina (AAS) em altas doses apresenta efeito anti-inflamatório e em baixas doses inibição da agregação plaquetária em crianças com Kawasaki; assim, na fase aguda a aspirina é utilizada na dose de $80-100 \mathrm{mg} / \mathrm{kg} /$ dia dividida em quatro tomadas diárias para potencializar o efeito anti-inflamatório da IGIV, porém não diminui a frequência de anormalidades coronarianas. ${ }^{2} \mathrm{O}$ tempo de uso da aspirina em altas doses varia entre as instituições; muitos centros hospitalares reduzem a dose após período de 48 a 72 horas de estado afebril, enquanto outros serviços mantêm o AAS por 14 dias em altas doses, estendendo-o por mais 48 ou 72 horas depois de cessada a febre. Em seguida, é feita a diminuição da dose da aspirina a $3-5 \mathrm{mg} / \mathrm{kg} / \mathrm{dia}$ e mantida por período de seis a oito semanas a partir do início da doença (nível $\mathrm{C}$ de evidência). Para crianças com anormalidades cardíacas, a aspirina é mantida indefinidamente (nível $\mathrm{B}$ de evidência). ${ }^{2,3}$

Não se deve administrar ibuprofeno concomitante à aspirina uma vez que o primeiro antagoniza a inibição plaquetária irreversível da aspirina. ${ }^{74}$ Outro cuidado importante em relação à criança em uso de aspirina em altas doses diz respeito à possibilidade de síndrome de Reye caso se apresente infecção por varicela ou influenza. Os pais devem ser orientados a procurar o pediatra caso a criança apresente qualquer dessas infecções em vigência do uso de salicilatos. ${ }^{2,3}$

Os corticosteróides têm sido usados nos casos em que os pacientes não responderam ao tratamento inicial com IGIV mais aspirina. ${ }^{75}$ Não obstante, os resultados após uso de corticóides são controversos. Um estudo randomizado, multicêntrico, placebo-controlado e duplo-cego mostrou que o corticóide reduz os níveis plasmáticos das proteínas de fase aguda (VHS e proteína $\mathrm{C}$ reativa), mas não diminui risco das anormalidades cardíacas, tempo de internação hospitalar, duração da febre e número de eventos adversos. ${ }^{76}$

Os estudos recomendam o uso de corticóide em pacientes que tomaram duas doses de IGIV sem resposta (Kawasaki refratário) clínica. O regime mais utilizado é o de pulsoterapia com metilprednisolona $30 \mathrm{mg} / \mathrm{kg} /$ dia infundida em duas ou três horas uma vez ao dia durante três dias.

Aproximadamente 10\% dos pacientes com DK não respondem à infusão da primeira dose de IGIV, ou seja, há persistência da febre ou sua recrudescência 36 horas após a primeira infusão de IGIV; esses pacientes são "não respondedores" à terapia inicial e apresentam maior risco de alterações coronarianas. Nesses casos os estudiosos recomendam o retratamento, isto é, a segunda dose de IGIV de $2 \mathrm{~g} / \mathrm{kg}$ em dose única. (nível de evidência C). ${ }^{77,78}$ Alguns centros realizam a terceira dose de IGIV, porém com resposta clínica variável - a febre pode persistir. ${ }^{79}$

Não há protocolos direcionados para o tratamento de DK refratária. Outros medicamentos têm sido utilizados nesses casos com resultados favoráveis, mas todos com base em relatos de casos ou estudos não randomizados e não controlados. Há relatos de tratamento com plasmaférese e efetiva resposta clínica ao diminuir a incidência de aneurismas coronarianos. ${ }^{80}$ No entanto, em razão dos riscos vigentes, a plasmaférese não é recomendada frequentemente (nível de evidência C).

Também são utilizados imunossupressores como ciclofosfamida ou ciclosporina associados à prednisona ou metilprednisolona com melhora da febre, porém sem alterar as anormalidades cardíacas. ${ }^{42,78,79}$ Os agentes citotóxicos apresentam efeitos adversos importantes e deve-se avaliar a relação custo/benefício em uma criança com DK e alteração cardiológica. Há relato de uso de metotrexato (10mg/superfície corpórea $1 \mathrm{x} / \mathrm{semana}$ ) com efetividade clínica - cessação da febre e sem progressão da dilatação de artérias coronarianas. ${ }^{81,82}$

No Japão já foi utilizada a ulinastatina, glicoproteína que inibe a elastase de neutrófilos, em alguns casos de DK refratária, porém seus resultados precisam ser comprovados. $\mathrm{O}$ abciximab, inibidor do receptor de glicoproteína IIb/IIIa plaquetário, tem sido utilizado nas fases aguda/subaguda de 
doentes com aneurismas grandes, com boa regressão no diâmetro do aneurisma, sugerindo que tal medicamento promova remodelamento vascular. ${ }^{2,83}$ Uma nova classe de agentes biológicos está sendo administrada, dentre os quais destacamos o infliximab (Remicade ${ }^{\circledR}$ ), anticorpo monoclonal contra o fator de necrose tumoral alfa (TNF- $\alpha$ ), com resultados parciais, nos poucos casos tratados, de melhora da febre e dos aneurismas. Não obstante, há necessidade de estudos controlados para avaliar e confirmar as indicações de seu uso. ${ }^{79,84}$

O tratamento nas fases subaguda e de convalescença de pacientes com aneurismas coronarianos visa prevenir a trombose (ativação plaquetária) e estenose do vaso. A aspirina em doses baixas (3-5 $\mathrm{mg} / \mathrm{kg} / \mathrm{dia})$ é a principal terapêutica para crianças com pequenos e médios aneurismas. ${ }^{2,3,6,42}$ Outros agentes antiplaquetários também são utilizados (clopidogrel, ticlopidina, dipiridamol) e, associados à aspirina, têm-se mostrado mais efetivos em bloquear a agregação plaquetária. ${ }^{2,3,42}$

Estudos randomizados são necessários para estabelecer o papel de agentes como a heparina de baixo

\section{REFERÊNCIAS}

1. Brandt HRC, Arnone M, Valente NYS, Criado PR, Sotto MN. Vasculite cutânea de pequenos vasos: etiologia, patogênese, classificação e critérios diagnósticos Parte I. An Bras Dermatol. 2007;82:387-406.

2. Newburger JW, Takahashi M, Gerber MA, Gewitz MH, Tani LY, Burns JC, et al. Diagnosis, Treatment, and LongTerm Management of Kawasaki Disease: A Statement for Health Professionals from the Committee on Rheumatic Fever, Endocarditis and Kawasaki Disease,Council on Cardiovascular Disease in the Young, American Heart Association. Pediatrics. 2004;114:1708-33.

3. Kim DS. Kawasaki disease. Yonsei Med J. 2006;47:759-72.

4. Rowley AH, Shulman ST. Kawasaki syndrome. Clin Microbiol Rev.1998;11:405-11.

5. Kushner HI, Bastian JF, Turnner CL and Burns JC. The Two Emergences of Kawasaki Syndrome and the Implications for the Developing World. Pediatr Infect Dis J. 2008;27:377-83.

6. Chung CJ, Stein L. Kawasaki disease: A review. Radiology. 1998;208:25-33.

7. Dajani AS, Taubert KA, Gerber MA, Shulman ST, Ferrieri P, Freed M, et al. Diagnosis and therapy of Kawasaki disease in children. Circulation. 1993;87:1776-80.

8. Satou GM, Giamelli J, Gewitz MH. Diagnosis, management, and long-term implications. Cardiol Rev. 2007;15:163-9.

9. Park YW, Han JW, Park IS, Kim CH, Cha SH, Ma JS, et al. Kawasaki disease in Korea, 2003-2005. Pediatr Infect Dis J. 2007;26:821-3.

10. Esper F, Shapiro ED, Weibel C, Ferguson D, Landry ML, peso molecular, warfarin e anticorpos monoclonais contra o receptor IIb/IIIa plaquetário no manejo das crianças com aneurismas gigantes. $\mathrm{O}$ mais comum regime antitrombótico para pacientes com aneurisma gigante é a aspirina em baixas doses associada ao warfarin com objetivo de manter o INR (international normalized ratio) entre dois e 2,5 (nível de evidência $\mathrm{C}$ ). ${ }^{2,85}$

\section{CONCLUSÃO}

A doença de Kawasaki é vasculite sistêmica com manifestação cutânea através de exantema polimórfico e, em virtude disso, deve fazer parte do conhecimento do dermatologista como diagnóstico diferencial de exantemas virais e escarlatina. A provável consequência da vasculite é desencadear a formação de aneurismas coronarianos com os riscos inerentes de obstrução e estenose desses vasos e aumento de morbimortalidade. O principal desafio do médico é a suspeição diagnóstica precoce, uma vez que o início do tratamento com IGIV nos primeiros 10 dias altera a história natural da doença.

Kahn JS. Association between a novel human coronavirus and Kawasaki Disease. J Infect Dis. 2005; 191:499-502.

11. Rowley AH, Shulman ST. New developments in the search for the etiologic agent of Kawasaki disease. Curr Opin in Pediatr. 2007;19:71-4.

12. Leung DYM, Giorno RC, Kazem LV, Flynn PA, Busse JB. Evidence for superantigen involvement in cardiovascular injury due to Kawasaki syndrome. J Immunol. 1995;55:5018-21.

13. Proft T, Fraser JD. Bacterial superantigens. Clin Exp Immunol. 2003;133:299-306.

14. Llewelyn M, Cohen J. Superantigens: microbial agents that corrupt immunity. Lancet Infect Dis. 2002;2:156-62.

15. Matsubara K, Fukaya T. The role of superantigens of group A Streptococcus and Staphylococcus aureus in Kawasaki disease. Curr Opin Infect Dis. 2007;20:298-303.

16. Leung DY, Meissner HC, Shulman ST, Mason WH, Gerber MA, Glode MP, et al. Prevalence of superantigensecreting bacteria in patients with Kawasaki Disease. J Pediatr. 2002;140:742-6.

17. Rowley AH, Shulman ST, Spike BT, et al. Oligoclonal IgA response in the vascular wall in acute Kawasaki disease. J Immunol. 2001;166:1334-43.

18. Rowley AH, Baker SC, Shulman ST, Mask CA, Baker SC. Cytoplasmic inclusion bodies are detected by synthetic antibody in ciliated bronchial epithelium during acute Kawasaki Disease. J Infect Dis. 2005;192:1757-66.

19. Caquard M, Parlier G, Siret D. [Family observation of Kawasaki disease: 2 cases in sister and brother]. Arch Pediatr. 2006;13:453-55. 
20. Burns JC, Shimizu C, Shike H. Candidate genes for susceptibility to Kawasaki disease (KD): analysis of preferentially transmitted alleles in patient/ parent triads. Pediatr Res. 2003;53:1857A. (abstract)

21. Breunis WB, Biezeveld MH, Geissler J, Kuipers IM, Lam $\mathrm{J}$, Ottenkamp J, et al. Polymorphisms in chemokine receptor genes and susceptibility to Kawasaki disease. Clin Exp Immunol. 2007;150:83-90.

22. Wu SF, Chang JS, Wan L, Tsai CH, Tsai FJ. Association of IL-1Ra gene polymorphism, but no association of IL-1beta and IL- 4 gene polymorphisms, with Kawasaki disease. J Clin Lab Anal. 2005;19:99-102.

23. Hui-Yuen JS, Duong TT, Yeung RS. TNF-alpha is necessary for induction of coronary artery inflammation and aneurysm formation in an animal model of Kawasaki disease. J Immunol. 2006;176:6294-301.

24. Lau AC, Duong TT, Ito S, Yeung RSM. Matrix Metalloproteinase 9 Activity Leads to Elastin Breakdown in an Animal Model of Kawasaki Disease. Arthritis Rheum. 2008;58:854-63.

25. Ikeda $\mathrm{K}$, Ihara $\mathrm{K}$, Yamaguchi $\mathrm{K}$, Muneuchi J, Ohno T, Mizuno Y, et al. Genetic Analysis of MMP Gene Polymorphisms in Patients With Kawasaki Disease. Pediatr Res. 2008;63:182-5.

26. Wittkowski H, Hirono K, Ichida F, Vogl T, Ye F, Yanlin X, et al. Acute Kawasaki Disease Is Associated With Reverse Regulation of Soluble Receptor for Advance Glycation End Products and Its Proinflammatory Ligand S100A12. Arthritis Rheum. 2007;56:4174-81.

27. Burns JC. S100 Proteins in the Pathogenesis of Kawasaki Disease. J Am Coll Cardiol. 2006;48:1265-7.

28. Muta H, Ishii M, Iemura M, Suda K, Nakamura Y, Matsuishi T. Effect of Revision of Japanese Diagnostic Criterion for fever in Kawasaki on Treatment and Cardiovascular Outcome. Circ J. 2007;71:1791-3.

29. Witt MT, Minich LL, Bohnsack JF, Young PC. Kawasaki disease: More Patients Are Being Diagnosed Who Do Not Meet American Heart Association Criteria. Pediatrics. 1999;104:1-10.

30. Yeung RSM. Pathogenesis and treatment of Kawasaki's disease. Curr Opin Rheumatol. 2005;17:617-23.

31. Yanagi S, Nomura Y, Masuda K, Koriyama C, Sameshima $\mathrm{K}$, Eguchi T, et al. Early diagnosis of Kawasaki disease in patients with cervical linphadenopathy. Pediatr Int. 2008;50:179-83.

32. Chakrabartty S, Pramanik S,Thapa R. Difficulties in the Diagnosis of Kawasaki Disease. Indian Pediatr. 2006;43:728-31.

33. Bastian JF and Kushner HI. Diagnosing Kawasaki syndrome. Rheumatology (Oxford). 2006;45:240-1.

34. Kawasaki T. General review and problems in Kawasaki Disease. Jpn Heart J. 1995; 36:1-12.

35. Cassidy JT, Petty RE. Vasculitis. In: Cassidy JT, Petty RE, eds. Textbook of pediatric rheumatology. 3rd ed. Philadelphia, W.B: Saunders Company; 1995. p. 365-422.

36. Narayanan SN, Krishnave NI, Sabarinathan K. Kawasaki disease. Indian Pediatr. 1997;34:139-43.

37. Ulloa-Gutierrez R, Acón-Rojas F, Camacho-Badilla K, Soriano-Fallas A. Pustular rash in Kawasaki syndrome. Pediatr Infect Dis J. 2007;26:1163-5.
38. Kwan YW, Leung CW. Pustulo-vesicular skin eruption in a child with probable Kawasaki disease. Eur J Pediatr. 2005; 164:770-1.

39. Wolff AE, Hansen KE, Zakowski L. Acute Kawasaki Disease: not just for kids. J Gen Intern Med. 2007;22:681-4.

40. Rozo JC, Jefferies JL, Eidem BW, Cook PJ. Kawasaki disease in the adult: a case report and review of the literature. Tex Heart Inst J. 2004;31:160-4.

41. Sève P, Stankovic K, Smail A, Durand DV, Marchand G, Broussolle C. Adult Kawasaki Disease: report of two cases and literature review. Semin Arthritis Rheum. 2005;34:785.

42. Pinna GS, Kafezis DA, Tselkas OI, Skevaki CL. Kawasaki disease: an overview. Curr Opin Infect Dis. 2008; 21: 263-70.

43. Muise A, Tallett SE, Silverman ED. Are children with Kawasaki Disease and prolonged fever at risk for Macrophage Activation Syndrome? Pediatrics. 2003;112(Pt 1):495-7.

44. Moran AM, Newburger JW, Sanders SP, Parness IA, Spevak PJ, Burns JC, et al. Abnormal myocardial mechanics in Kawasaki disease: rapid response to gamma-globulin. Am Heart J. 2000;139:217-23.

45. Falcini F. Kawasaki disease. Curr Opin Rheumatol. 2006;18:33-8.

46. Kato H, Sugimura T, Akagi T et al. Long-term consequences of Kawasaki disease: a 10 to 21 year follow-up study of 594 patients. Circulation. 1996;94:1379-85.

47. Newburger JW, Fulton DR. Kawasaki disease. Curr Opin Pediatr. 2004;16:508-14.

48. Nakamura Y, Yashiro M, Uehara R, Watanabe M, Tajimi M, Oki I, et al. Use of laboratory data to identify risk factors of giant coronary aneurysms due to Kawasaki disease. Pediatr Int. 2004;46:33-8.

49. Takemura A, Suzuki A, Kitazume T, Sonobe T, Tsuchiya $\mathrm{K}$, Korenaga T. [The utility of coronary magnetic resonance angiography in children under six years of age with Kawasaki disease.] Nippon Hoshasen Gijutsu Gakkai Zasshi. 2008;64:874-6. (Abstract in English and the article in japanese)

50. Burns JC. The riddle of Kawasaki disease. N Engl J Med 2007;356:659-61.

51. Serkan G, Arda S, Ercan T, Cizmeli MO. Coronary artery evaluation in Kawasaki disease by dual source multidetector CT coronary angiography in children. Anadolu Kardiyol Derg. 2008;8:8-14.

52. Nolan TE, Hankins GD. Myocardial infarction in pregnancy. Clin Obstet Gynecol. 1989;32:68-75.

53. Tsuda E, Ishihara Y, Kawamata K, Tsukano S, Negi R, Echigo S, et al. Pregnancy and delivery in patients with coronary artery lesions caused by Kawasaki disease. Heart. 2005;91:1481-2.

54. Patrizi A, Raone B, Savoia F, Ricci G, Neri I. Recurrent toxin-mediated perineal erythema: eleven pediatric cases. Arch Dermatol. 2008;144:239-43.

55. Kato H, Ichinose E, Kawasaki T. Myocardial infarction in Kawasaki disease: clinical analyses in 195 cases. J Pediatr . 1986;108:923-7.

56. Baker AL, Newburger JW. Cardiology patient pages. Kawasaki disease. Circulation. 2008;118:110-2. 
57. Sugimura T, Kato H, Inoue O, Takagi J, Fukuda T, Sato $\mathrm{N}$. Vasodilatory response of the coronary arteries after Kawasaki disease: evaluation by intracoronary injection of isosorbide dinitrate. J Pediatr. 1992;121 (5 Pt 1):684-8.

58. Iemura M, Ishii $M$, Sugimura $T$, Akagi $T$, Kato H. Long term consequences of regressed coronary aneurysms after Kawasaki disease: vascular wall morphology and function. Heart. 2000;83:307-11.

59. Burns JC, Shike H, Gordon JB, Malhotra A, Schoenwetter M, Kawasaki T. Sequelae of Kawasaki disease in adolescents and young adults. J Am Coll Cardiol. 1996;28:253-7.

60. Muzik O, Paridon SM, Singh TP, Morrow WR, Dayanikli F, Di Carli MF. Quantification of myocardial blood flow and flow reserve in children with a history of Kawasaki disease and normal coronary arteries using positron emission tomography. J Am Coll Cardiol. 1996; 28:757-62.

61. Nakamura Y, Aso E, Yashiro M, Uehara R, Watanabe M, Oki I, et al. Mortality Among Persons With a History of Kawasaki Disease in Japan. Circ J. 2008;72:134-8.

62. Tsuji T, Suzuki J, Shimamoto R, Yamazaki T, Ohomoto Y, Iwasawa K, et al. Morbidity Prevalence Rate of Kawasaki Disease Assessed by Single Cross-Sectional HistoryTaking. Int Heart J 2007; 48:615-21.

63. Tse SML, Silverman ED, McGrindle BW, Yeung RSM. Early treatment with intravenous immunoglobulin in patients with Kawasaki disease. J Pediatr. 2002; 140:450-5.

64. Durongpisitkul K, Gururaj VJ, Park JM, Martin CF. The prevention of coronary artery aneurysm in Kawasaki disease: a meta-analysis on the efficacy of aspirin and immunoglobulin treatment. Pediatrics. 1995; 96: 1057-61.

65. Terai M, Shulman ST. Prevalence of coronary artery abnormalities in Kawasaki disease is highly dependent on gamma globulin dose but independent of salicylate dose. J Pediatr. 1997;131:888-93.

66. Lee KY, Lee HS, Hong JH, Han JW, Lee JS, Whang KT. High-dose intravenous immunoglobulin downregulates the activated levels of inflammatory indices except erythrocyte sedimentation rate in acute stage of Kawasaki disease. J Trop Pediatr. 2005;51:98-101.

67. Wilson N, Heaton P, Calder L, Nicholson R, Stables S, Gavin R. Kawasaki disease with severe cardiac sequelae: lessons from recent New Zealand experience. J Paediatr Child Health. 2004;40(9-10):524-9.

68. Abe J, Jibiki T, Noma S, Nakajima T, Saito H, Terai M. Gene expression profiling of the effect of highdose intravenous Ig in patients with Kawasaki disease. J Immunol. 2005;174:5837-45.

69. Sugita K, Hirao J, Arisaka O, Eguchi M. Gamma-globulin-induced modulation with necrotic-like morphology of peripheral blood neutrophils. Eur J Pharmacol. 2005; 513:141-4.

70. Muta H, Ishii M, Egami K, Furui J, Sugahara Y, Akagi T, et al. Early intravenous gamma-globulin treatment for Kawasaki disease: the nationwide surveys in Japan. J Pediatr. 2004;144:496-9.
71. Fong NC, Hui YW, Li CK, Chiu MC. Evaluation of the efficacy of treatment of Kawasaki disease before day 5 of illness. Pediatr Cardiol. 2004;25:31-4.

72. Vecchiett G, Kerl K, Prins C, Kaya G, Saurat JH, French LE. Severe eczematous skin reaction after high-dose intravenous immunoglobulin infusion. Arch Dermatol. 2006;142:213-7.

73. Pickering LK, ed. Red Book. 2003 Report of the Committee on Infectious Diseases. Chicago, IL: American Academy of Pediatrics; 2003. p.74-5.

74. Catella-Lawson F, Reilly MP, Kapoor SC, Cucchiara AJ, DeMarco S, Tournier B, et al. Cyclooxygenase inhibitors and the antiplatelet effects of aspirin. N Engl J Med. 2001;345:1809-17.

75. Shulman ST. Is there a role for corticosteroids in Kawasaki disease? J Pediatr. 2003;142:601-3.

76. Newburger JW, Sleeper L A, McCrindle BW, Minich LL, Gersony W, Vetter VL, et al. Randomized Trial of Pulsed Corticosteroid Therapy for Primary Treatment of Kawasaki Disease. N Engl J Med. 2007;356:663-75.

77. Durongpisitkul K, Soongswang J, Laohaprasitiporn D, Nana A, Prachuabmoh C, Kangkagate C. Immunoglobulin failure and retreatment in Kawasaki disease. Pediatr Cardiol. 2003;24:145-8.

78. Wallace CA, French JW, Kahn SJ, Sherry DD. Initial intravenous gammaglobulin treatment failure in Kawasaki disease. Pediatrics. 2000;105:E-78

79. Freeman AF, Shulman ST. Refractory Kawasaki disease. Pediatric Infect Dis J 2004;23:463-4.

80. Imagawa $\mathrm{T}$, Mori $\mathrm{M}$, Miyamae $\mathrm{T}$, Ito $\mathrm{S}$, Nakamura $\mathrm{T}$, Yasui $\mathrm{K}$, et al. Plasma exchange for refractory Kawasaki disease. Eur J Pediatr. 2004;163:263-4.

81. Ahn SY, Kim DS. Treatment of intravenous immunoglobulin-resistant Kawasaki disease treated with methotrexate. Scand J Rheumatol. 2005;34:136-9.

82. Lee MS, Anh SY, Jang GC, Kim DS. A case of intravenous immunoglobulin-resistant Kawasaki disease treated with methotrexate. Yonsei Med J. 2002;43:527-32.

83. Williams RV, Wilke VM, Tani LY, Minich LL. Does abciximab enhance regression of coronary aneurysms resulting from Kawasaki disease? Pediatrics. 2002;109:109-14.

84. Oishi $T$, Fujieda $M$, Shiraishi $T$, Ono $M$, Inoue $K$, Takahashi A, et al. Infliximab treatment for refractory Kawasaki disease with coronary artery aneurysm. Circ J. 2008;72:850-2.

85. Bradley DJ, Glode MP. Kawasaki disease: the mystery continues. West J Med. 1998; 168:23-29.

ENDEREÇO PARA CORRESPONDÊNCIA / MAILING ADDRESS:
Patrícia Aparecida de Castro
Av. Parque Águas Claras lote 2615
edifício Magnólia apartamento 906
71906500 Águas Claras - Brasília DF
Tel./Fax: $619200-0772$ / 61 3536-1839
e-mail:patricia00castro@yaboo.com.br

ENDEREÇO PARA CORRESPONDÊNCIA / MAILING ADDRESS:

Av. Parque Águas Claras lote 2615

edifício Magnólia apartamento 906

71906500 Águas Claras - Brasília DF

e-mail:patricia0ocastro@yaboo.com.br 